Efficient search termination without task experience

\author{
Matan Mazor ${ }^{1} \&$ Stephen M. Fleming ${ }^{1,2,3}$ \\ ${ }^{1}$ Wellcome Centre for Human Neuroimaging, UCL \\ ${ }^{2}$ Max Planck UCL Centre for Computational Psychiatry and Ageing Research \\ ${ }^{3}$ Department of Experimental Psychology, UCL
}

Author Note: Anonymized data, analysis scripts, and stimulus materials are available at github.com/matanmazor/termination. Pre-registrations are available at osf.io/fyjcp/. The authors have no conflicting interests to disclose. A previous version of this paper appears on PsyArxiv. This research was funded in whole, or in part, by the Wellcome Trust [Grant number 203147/Z/16/ ]. For the purpose of open access, the author has applied a CC BY public copyright licence to any Author Accepted Manuscript version arising from this submission. Correspondence concerning this article should be addressed to Matan Mazor, 12 Queen Square, London WC1N 3BG. E-mail: mtnmzor@gmail.com

Acknowledgements: We thank Todd Horowitz and the two anonymous reviewers for their insightful comments and suggestions. The Wellcome Centre for Human Neuroimaging is supported by core funding from the Wellcome Trust (203147/Z/16/Z). S.M.F. is supported by a Sir Henry Dale Fellowship jointly funded by the Wellcome Trust and the Royal Society (206648/Z/17/Z), and a Philip Leverhulme Prize from the Leverhulme Trust. 


\begin{abstract}
As a general rule, if it is easy to detect a target in a visual scene, it is also easy to detect its absence. To account for this, models of visual search explain search termination as resulting either from counterfactual reasoning over second-order representations of search efficiency, automatic extraction of ensemble statistics of a display, or heuristic adjustment of a search termination strategy based on previous trials. Traditional few-subjects/many-trials lab-based experiments render it impossible to disentangle the unique contribution of these different processes to absence pop-out - the immediate recognition that a feature is missing from a display. In two pre-registered large-scale online experiments $(\mathrm{N} 1=1187, \mathrm{~N} 2=887)$ we show that search termination times are already aligned with target identification times in the very first trials of the experiment, before any experience with target presence. Exploratory analysis reveals that explicit metacognitive knowledge about search efficiency is not necessary for efficient search termination. We conclude that for basic stimulus properties, efficient inference about absence is independent of task experience, and of explicit metacognitive knowledge about visual search.
\end{abstract}




\section{Efficient search termination without task experience}

\section{$1 \quad$ Introduction}

Searching for the only blue letter in an array of yellow letters is easy, but searching for the only blue X in an array of yellow Xs and blue Ts is much harder (A. M. Treisman \& Gelade, 1980). This difference manifests in the time taken to find the target letter, but also in the time taken to conclude that the target letter is missing. In other words, easier searches not only make it easier to detect the presence of a target, but also to infer its absence. Differences in the speed of detecting the presence of a target have been attributed to pre-attentional mechanisms (A. M. Treisman \& Gelade, 1980) and guiding signals (J. Wolfe, 2021; J. M. Wolfe \& Gray, 2007) that can sometimes make the target item 'pop out' immediately without any attentional effort. In target-absent trials, however, there is nothing in the display to pop out. This raises a fundamental question: what makes some decisions about target absence easier than others?

Models of search termination offer three classes of answers to this question, based on counterfactual reasoning, ensemble perception, and task heuristics. According to counterfactual models, decisions about target absence are guided by prior beliefs about search efficiency ("If it were present, I would have found the red book by now"). These comprise beliefs about regularities in the environment ("it it were present, the book would have been on this shelf"), and second-order beliefs about one's own perception and attention ("the red cover would have immediately drawn my attention"). We use the term second-order to refer to an agent's belief about their own cognition and perception (Fleming, Dolan, \& Frith, 2012). In recent versions of the Guided Search model (J. Wolfe, 2021; J. M. Wolfe, 2012), for example, search termination is triggered by a noisy quitting signal accumulator reaching a quitting threshold, which can be 
adapted to maximize long-time search efficiency, and be affected by prior second-order beliefs about the effects of set size and crowding on search difficulty (J. M. Wolfe, 2012). Similarly, in Competitive Guided Search, the probability of terminating a search is a function of several factors, including a free parameter that indexes beliefs about finding a hypothetical target, had it been present (Moran, Zehetleitner, Müller, \& Usher, 2013). Finally, in a fixation-based model of visual search, the number of items that are concurrently scanned within a single fixation (the functional visual field) depends on the expected difficulty of finding a hypothetical target: with more items for easy searches and fewer items for more difficult ones (Hulleman \& Olivers, 2017).

Ensemble perception accounts of visual search postulate that some global properties of a display can be extracted without allocating attention to individual items, and that in some cases these global properties are sufficient to conclude that a target is absent. For example, according to Feature Integration Theory, pre-attentive activation in feature maps can provide participants with information about the presence or absence of a feature in the display (A. M. Treisman \& Gelade, 1980). In Treisman's updated models, attention can be deployed to sets of items, resulting in the extraction of global scene statistics (A. Treisman, 2006). The absence of a relevant feature, or the perception of the uniformity of an array of objects, is then sometimes sufficient to make an immediate 'target absent' decision, without processing any individual stimulus.

Finally, heuristic-based models suggest that quitting parameters are acquired by participants as they perform a task, sometimes by following very simple rules. For example, in one model, an internal activation threshold decreases following incorrect and increases following correct 'no' responses (Chun \& Wolfe, 1996). A higher activation threshold results in the scanning of less distractors, giving rise to shorter search times for easier searches. This simple 
heuristic provides an excellent fit to data from a visual search task with hundreds of trials, and does so without requiring that subjects hold any prior knowledge or expectations about search efficiency.

In traditional visual search experiments, where participants perform hundreds of trials of similar searches, it is impossible to disentangle the contributions of these three putative mechanisms to search termination. Yet, the three accounts make different predictions for the earliest trials of a visual search experiment, where participants encounter the stimuli for the first time. In these trials, quitting time cannot reflect the adaptive adjustment of a threshold based on previous trials, or the statistical learning of regularities in the experiment. Instead, efficient search termination without task experience must rely on an immediate perception of ensemble properties of the display, prior second-order knowledge about one's own search efficiency, or a combination of both.

In two pre-registered experiments we focus on feature search for colour and shape. Focusing on the first four trials of the task, we ask whether prior experience with the task and stimuli is necessary for efficient search termination in feature searches. Unlike typical visual search experiments that comprise hundreds or thousands of trials, here we collect only a handful of trials from a large pool of online participants. This unusual design allows us to reliably identify search time patterns in the first trials of the experiment, before ever experiencing finding the target. To anticipate our results, we find that efficient search termination for single features does not depend on task experience. In an exploratory analysis on a subset of participants, we further show that efficient search termination is also independent of explicit metacognitive knowledge about the task (that is, knowledge that is available for self-report). 


\section{Experiment 1}

In Experiment 1, we examined search termination in the case of colour search. When searching for a deviant colour, the number of distractors has virtually no effect on search time (colour pop-out; e.g., D’Zmura, 1991), for both 'target present' and 'target absent' responses. Here we asked whether efficient quitting in colour search (color absence pop-out) is dependent on task experience. A detailed pre-registration document for Experiment 1 can be accessed at osf.io/yh82v/.

\subsection{Participants}

The research complied with all relevant ethical regulations, and was approved by the Research Ethics Committee of University College London (study ID number 1260/003). 1187 Participants were recruited via Prolific, and gave their informed consent prior to their participation. They were selected based on their acceptance rate $(>95 \%)$ and for being native English speakers. Following our pre-registration, we collected data until we reached 320 included participants for each of our pre-registered hypotheses (after applying our pre-registered exclusion criteria). The entire experiment took around 3 minutes to complete (median completion time: 3.19 minutes). Participants were paid $£ 0.38$ for their participation, equivalent to an hourly wage of $£ 7.14$.

\subsection{Procedure}

A static version of Experiment 1 can be accessed on matanmazor.github.io/termination/experiments/demos/exp1/. Participants were first instructed about the visual search task. Specifically, that their task is to report, as accurately and quickly as possible, whether a target stimulus was present (press ' $J$ ') or absent (press ' $\mathrm{F}$ '). Then, to 
familiarize participants with the structure of the task and response requirements, practice trials were included. In practice trials set size was always 3 , the target stimulus was a rotated $\mathrm{T}$, and distractors rotated Ls. Using a spatial-configuration search task without varying set size between trials rendered practice trials completely uninformative about a set-size effect on search time, or the parallel versus serial nature of feature and conjunction searches. This ensured that efficient search termination on the first trials of the main task could not be due to learning in these first trials. Practice trials were delivered in short blocks of 6 trials each, and the main part of the experiment started only once participants responded correctly on at least five trials in a block (see Fig. 1). 

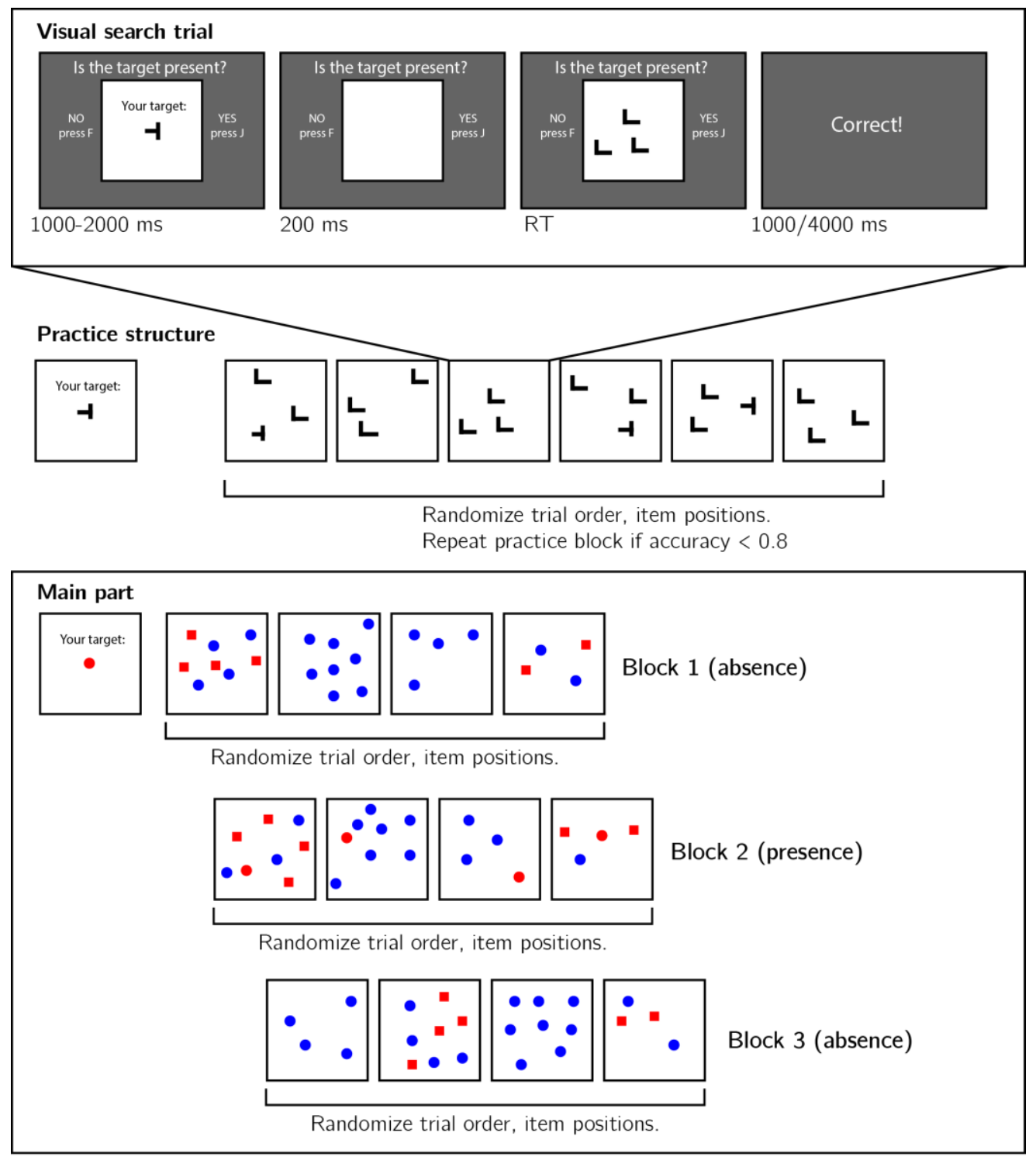

Figure 1. Experimental design. Top panel: each visual search trial started with a screen indicating the target stimulus. The search display remained visible until a response was recorded. To motivate accurate responses, the feedback screen remained visible for one second following correct responses and for four seconds following errors. Middle panel: after reading the instructions, participants practiced the visual search task in blocks of 6 trials, until they had reached an accuracy level of 0.83 correct or higher (at most one error in a block of 6 trials). Bottom panel: the main part of the experiment comprised 12 trials only, in which the target was a red dot. Unbeknown to subjects, only trials 5-8 (Block 2) were targetpresent trials, and the remaining trials were target-absent trials. Each 4-trial block followed a 2 by 2 design, with factors being set size (4 or 8 ) and distractor type (color or conjunction; blue dots only or blue dots and red squares, respectively). 
In the main part of the experiment, participants searched for a red dot among blue dots or a mixed array of blue dots and red squares. Set size was set to 4 or 8 , resulting in a 2-by-2 design (search type: color or color $\times$ shape, by set size: 4 or 8 ). Critically, and unbeknown to subjects, the first four trials were always target-absent trials (one of each set-size $\times$ search-type combination), presented in randomized order. These trials were followed by the four corresponding targetpresent trials, presented in randomized order. The final four trials were again target-absent trials, presented in randomized order.

\subsubsection{Randomization. To ensure pre-registration time-locking (in other words,} that pre-registration preceded data collection), we employed randomization-based preregistration. We used the SHA256 cryptographic hash function to translate our pre-registered protocol folder (link:

https://github.com/matanmazor/termination/blob/main/experiments/Experiment1/protocolFolder. zip) to a string of 256 bits (protocol sum:

eff9702fd40c04c703391c55a673a3b0a0719531bb1d2bfab2b813cbd90da227). These bits were then combined with the unique identifiers of single subjects, and the resulting string was used as seed for initializing the Mersenne Twister pseudorandom number generator prior to determining the order and timing of experimental events (link to relevant lines in experimental code). This way, experimental randomization was causally dependent on, and therefore could not have been determined prior to, the specific contents of our pre-registration document. (Mazor, Mazor, \& Mukamel, 2019).

\subsection{Data analysis}

\subsubsection{Rejection criteria.}

Participants were excluded for making more than one error in the main part of the experiment, or for having extremely fast or slow reaction times in 
one or more of the tasks (below 250 milliseconds or above 5 seconds in more than $25 \%$ of the trials).

Error trials, and trials with response times below 250 milliseconds or above 1 second were excluded from the response-time analysis. All pre-registered analyses without RT-based exclusion are reported in appendix A.

\subsubsection{Data preprocessing. To control for within-block trial order effects, a linear} regression model was fitted separately for each block and participant, predicting search time as a function of trial serial order within the block $\left(R T \sim \beta_{0}+\beta_{1} i\right.$, with $i$ denoting the mean-centered serial position within a block). Search times were corrected by subtracting the product of the slope and the mean-centered serial position, in a block-wise manner.

Subject-wise search slopes were then extracted for each combination of search type (color or conjunction) and block number by fitting a linear regression model to the reaction time data with one intercept and one set-size term.

\subsubsection{Hypotheses and analysis plan. Experiment 1 was designed to test several} hypotheses about the contribution of metacognitive knowledge to search termination, the state of this knowledge prior to engaging with the task, and the effect of experience on this metacognitive knowledge. The specifics of our pre-registered analysis can be accessed in the following link:

https://osf.io/ea385. We outline some possible search time patterns and their pre-registered interpretation in Fig. 2. 


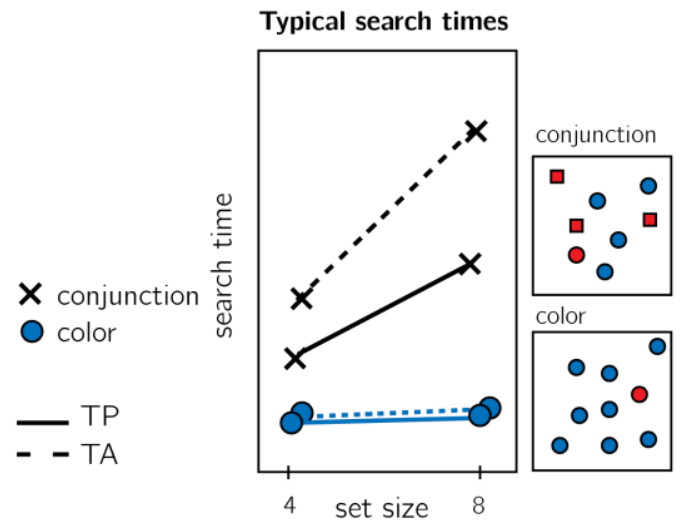

Positive trials only

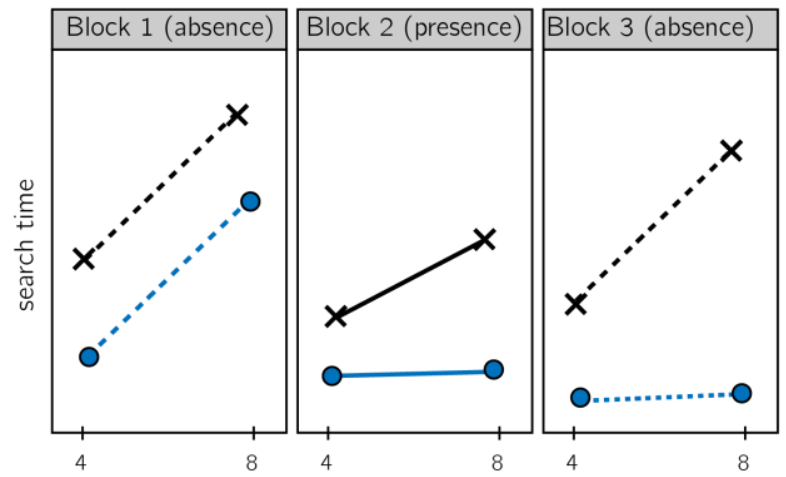

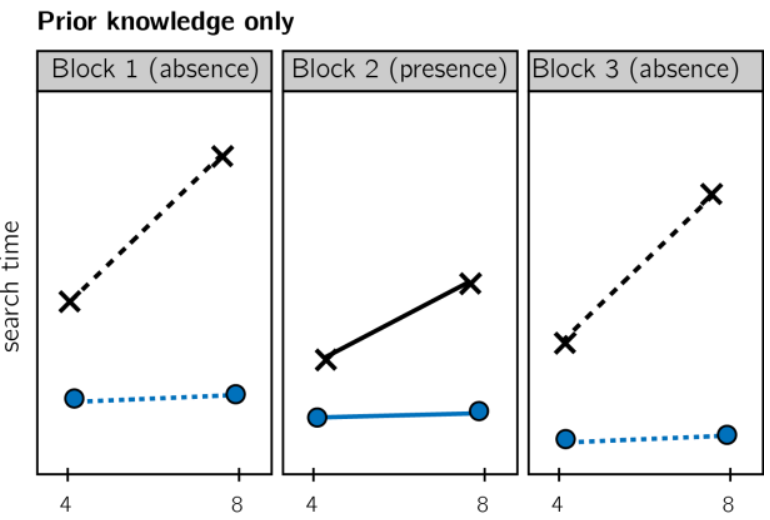

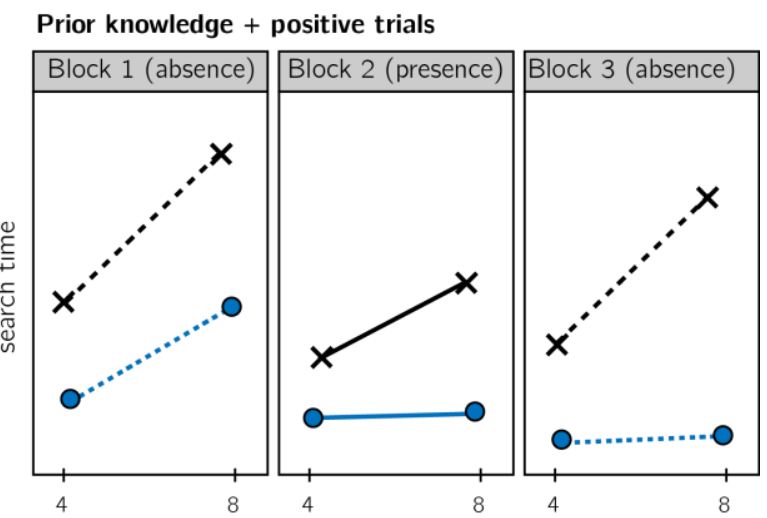

Figure 2. Visualization of Hypotheses. Top left: typical search times in visual search experiments with many trials (where TP $=$ Target Present responses; TA = Target Absent responses). Set size ( $x$ axis) affects search time in conjunction search, but much less so in color search. However, it is unclear whether this pattern also holds in the first target-absent trials in an experiment. Different models make different predictions about target-absent search times in the first block of the experiment. Top right: one possibility is that the same qualitative pattern will be observed in our design, with an overall decrease in response time as a function of trial number. This would suggest that the preconditions for efficient inference about absence were fully in place before engaging with the task. Bottom left: an alternative pattern is that the same qualitative pattern will be observed for blocks 2 and 3 , but not in block 1 . This would suggest that for inference about absence to be efficient, participants had to first experience some target-present trials. Bottom right: alternatively, some but not all preconditions for efficient inference about target absence may depend on exposure to target-present trials. This would manifest as different slopes for conjunction and color searches in blocks 1 and a learning effect for color search between blocks 1 and 3. 
Analysis comprised a positive control based on target-present trials, a test of the presence of a pop-out effect for target-absent color search in block 1, and a test for the change in slope for target-absent color search between blocks 1 and 3. All statistical tests were performed on search slopes, and were therefore orthogonal to effects of target prevalence or serial order on response speed and accuracy (Ishibashi, Kita, \& Wolfe, 2012; J. M. Wolfe, Horowitz, \& Kenner, 2005). In our pre-registration document, we identified 'pop-out' with searches that produce search slopes significantly lower than $10 \mathrm{~ms} /$ item in a one-sided t-test. We subsequently realized that this definition was flawed, as empirical distributions of search slopes do not show a clear cut-off between 'pop-out' and other searches (Wolfe, 1998). Instead, we now report in the main text the search slopes and their $95 \% \mathrm{CI}$, and the comparison with $10 \mathrm{~ms} /$ item is presented in the Appendix. All hypotheses were tested using a within-subject t-test, with a significance level of 0.05 .

Given the fact that we only have one trial per cell, one excluded trial is sufficient to make some hypotheses impossible to test on a given participant. For this reason, for each hypothesis separately, participants were included only if all necessary trials met our inclusion criteria. This meant that some hypotheses were tested on different subsets of participants.

\subsubsection{Transparency and Openness. We report how we determined our sample} size, all data exclusions (if any), all manipulations, and all measures in the study. We used R [Version 4.0.5; R Core Team (2019)] and the R-packages BayesFactor [Version 0.9.12.4.2; Morey and Rouder (2018)], cowplot [Version 1.1.1; Wilke (2019)], dplyr [Version 1.0.7; Wickham, François, Henry, and Müller (2020)], ggplot2 [Version 3.3.5; Wickham (2016)], jsonlite [Version 1.7.2; Ooms (2014)], lsr [Version 0.5; Navarro (2015)], MESS [Version 0.5.7; Ekstrøm (2019)], papaja [Version 0.1.0.9997; Aust and Barth (2020)], pwr [Version 1.3.0; 
Champely (2020)], and tidyr [Version 1.1.3; Wickham and Henry (2020)] for all our analyses. A detailed pre-registration document for Experiment 1 can be accessed at osf.io/yh82v/. All analysis scripts and anonymized data are available at github.com/matanmazor/termination.

\section{$2.4 \quad$ Results}

On average, participants performed 7.16 practice trials, and $86 \%$ of participants finished the practice part after one block of six trials. Overall mean accuracy in the main task was 0.95 $($ standard deviation $=0.06)$. Median reaction time was $623.98 \mathrm{~ms}$ (median absolute deviation $=$ 127.37). In all further analyses, only correct trials with response times between 250 and $1000 \mathrm{~ms}$ are included.

Hypothesis 1 (positive control): Search times in block 2 (target-present) followed the expected pattern, with a steep slope for conjunction search $(M=12.52,95 \%$ CI $[10.08,14.95])$ and a shallow slope for color search $(M=3.91,95 \%$ CI $[2.13,5.70]$; see middle panel in Fig. $3 \mathrm{~A})$. The difference between the slopes was significant $(t(749)=6.50, p<.001 ; \mathrm{d}=0.24)$. This positive control served to validate our method of using two trials per participant for obtaining reliable group-level estimates of search slopes.

Hypothesis 2: Our central focus was on results from block 1 (target-absent). Here participants didn't yet have experience with searching for the red dot. Similar to the second block, conjunction search slope was steep $(M=18.41,95 \%$ CI $[14.95,21.87])$. Search slopes for color absence were shallow $(M=0.15,95 \%$ CI $[-2.43,2.72]$, and significantly shallower than search slopes for conjunction search $(t(413)=6.55, p<.001 ; \mathrm{d}=0.32$; see leftmost panel in Fig. 3A), indicating that a color-absence pop-out is already in place prior to direct task 
experience. This result is in line with the prior-knowledge only model (see Fig. 2), in which participants have valid expectations for efficient color search, prior to engaging with a task.

Pre-registered hypotheses 3-5 were designed to test for a learning effect between blocks 1 and 3, before and after experience with observing a red target among blue distractors. Given the overwhelming pop-out effect for target-absent trials in block 1, not much room for additional learning remained. Indeed, results from these tests support a prior-knowledge only model.

Hypothesis 3: Like in the first block, in the third block color search slope was shallow $(M=2.27,95 \%$ CI $[0.36,4.17]$, and significantly different from the conjunction search slope $(t(745)=11.16, p<.001 ; d=0.41 ;$ see rightmost panel in Fig. $3 \mathrm{~A})$. This result is not surprising, given that a pop-out effect was already observed in block 1 .

Hypothesis 4: To quantify the learning effect for color search, we directly contrasted the search slope for color search in blocks 1 and 3. We find no evidence for a learning effect $(t(799)=-1.15, p=.250 ; \mathrm{d}=0.04)$. Furthermore, a Bayesian $\mathrm{t}$-test with a scaled Cauchy prior for effect sizes $(r=0.707)$ provided strong evidence in favour of the absence of a learning effect $\left(\mathrm{BF}_{01}=12.98\right)$

Hypothesis 5: In case of a learning effect for pop-out search, Hypothesis 5 was designed to test the specificity of this effect to color pop-out by computing an interaction between block number and search type. Given that no learning effect was observed, this test makes little sense. For completeness, we report that the change in slope between blocks 1 and 3 was similar for color and conjunction search $(M=-3.58,95 \%$ CI $[-10.52,3.36], t(320)=-1.01, p=.311$; $\mathrm{d}=0.06)$. 
A. Experiment 1
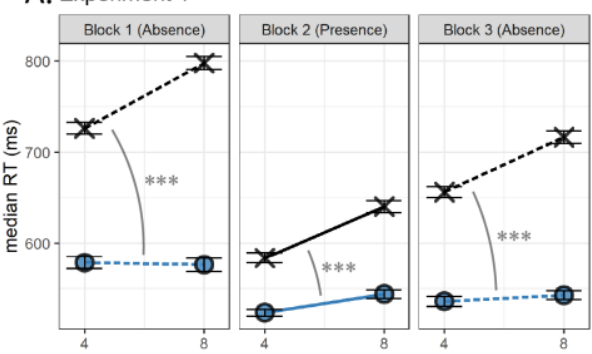
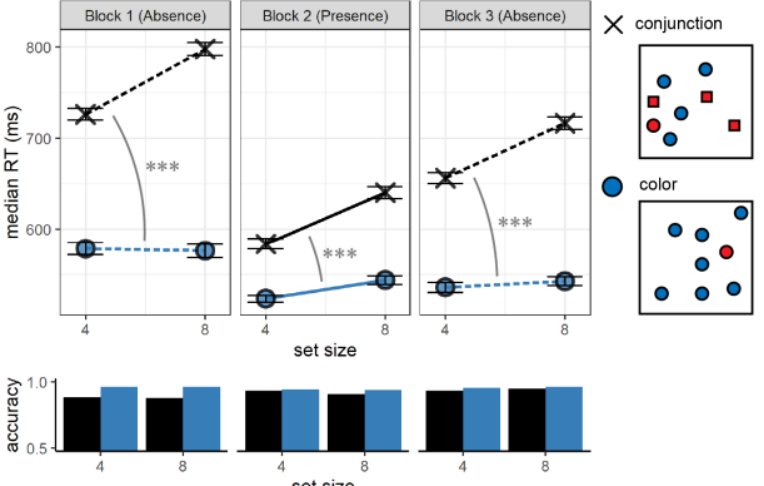

B. Experiment 2

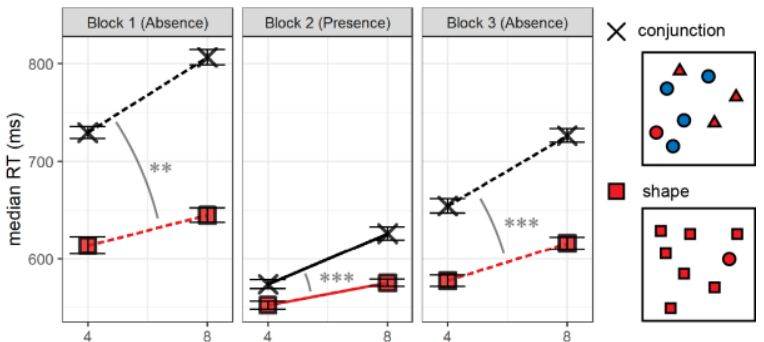

set size

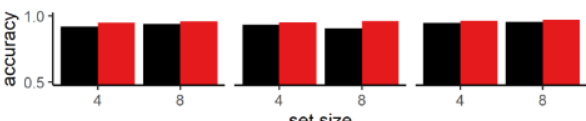

Figure 3. Main Results for Expeiments 1 (A) and 2 (B). Upper panel: median search time by distractor set size for the two search tasks across the three blocks (12 trials per participant). Correct responses only. Lower panel: accuracy as a function of block, set size and search type. Error bars represent the standard error of the median (estimated with bootstrapping). Significance stars correspond to the difference in slope between conjunction and feature search within a block. ${ }^{*}: p<0.5,{ }^{*}: p<0.01,{ }^{*} * *: p<0.001$

\subsection{Additional analysis: first trial only}

We considered the possibility that our results do not reflect true absence pop-out without task experience, but participants' ability to rapidly adjust their termination times based on feedback from previous trials, even within the four trials of the first block. To rule out such within-block learning effects, we tested whether participants showed a color-absence pop-out effect on the very first trial of the experiment. To this end, we analyzed first trial response times as a function of search type (conjunction or color) and set-size. Since these first trials were slower overall (median RT in the first trial: $881.30 \mathrm{~ms}$ compared to $630.34 \mathrm{~ms}$ in the last trial), for this exploratory analysis we did not exclude trials based on response times.

Even in this between-subject analysis, with only one trial per participant, we found a significant positive search slope for conjunction search $(42.75 \mathrm{~ms} / \mathrm{item}, p<0.01)$, but not for color search $(-12.27 \mathrm{~ms} / \mathrm{item}, p=.43)$. The difference in slopes between conjunction and color, quantified as the interaction between set size and search type in a two-way between-subject 
analysis of variance, was also significant $(F(1,1,041)=6.74, M S E=466,761.60, p=.010$, $\hat{\eta}_{G}^{2}=.006$; see Fig. 4A). In other words, a color-absence pop-out was already detectable in the very first trial of the experiment.
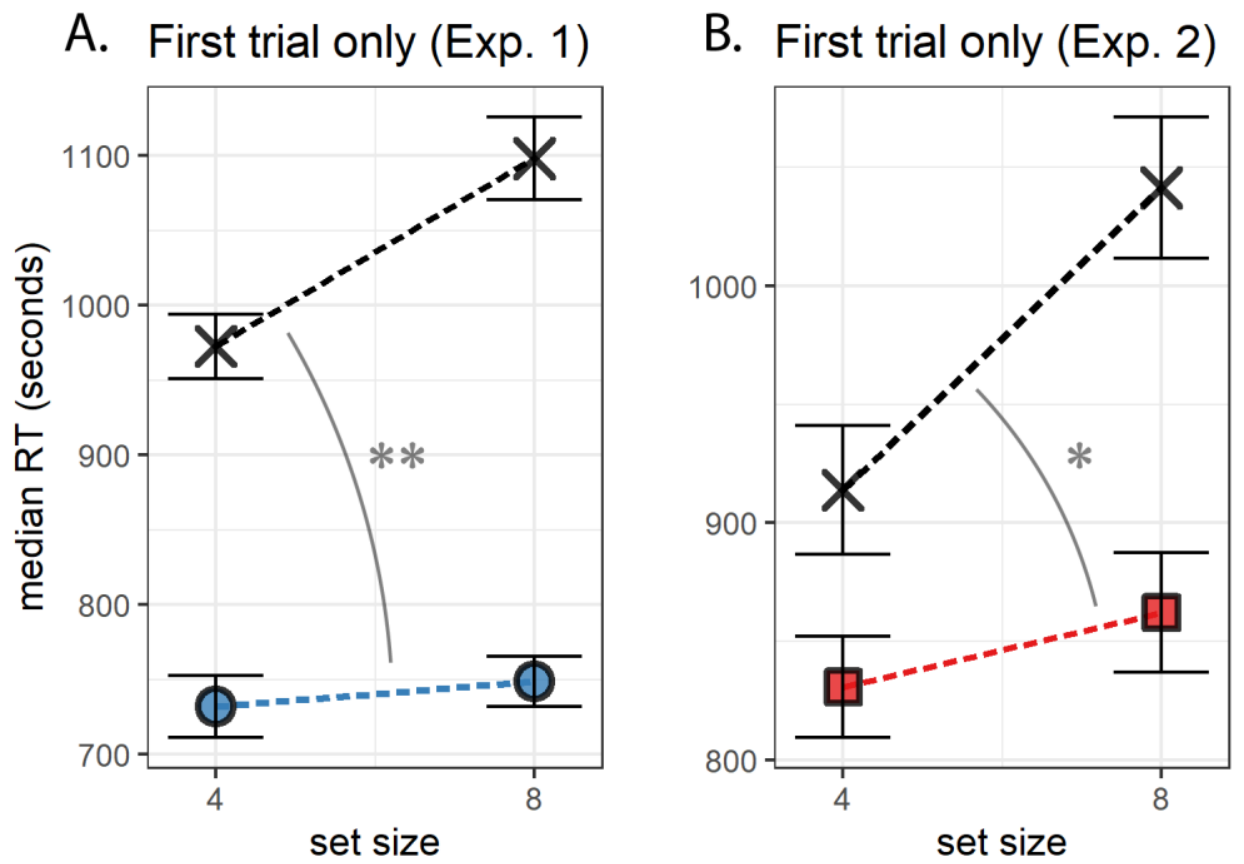

Figure 4. Median search time by distractor set size for Experiments 1 and 2, looking at the first trial of each participant only. Same conventions as in Fig. 3.

\section{$3 \quad$ Experiment 2}

Experiment 1 provided unequivocal evidence that color-absence pop-out occurs prior to experiencing color pop-out in the context of the same task. Experiment 2 was designed to extend these findings to another stimulus feature that is also found to efficiently guide attention: shape. Unlike colour space, which spans three dimensions only, the space of possible shapes is relatively unconstrained such that having prior knowledge of the expected effect of different shapes on attention might require a richer mental model of attentional processes. Furthermore, colour is agreed to be a 'guiding attribute of attention,' while it is unclear which shape features guide 
attention (J. M. Wolfe \& Horowitz, 2017). In this experiment we also included an additional control for prior experience with visual search tasks, and asked if knowledge about search efficiency is available for explicit metacognitive report.

\subsection{Participants}

The research complied with all relevant ethical regulations, and was approved by the Research Ethics Committee of University College London (study ID number 1260/003). 887 Participants were recruited via Prolific, and gave their informed consent prior to their participation. They were selected based on their acceptance rate $(>95 \%)$ and for being native English speakers. We collected data until we reached 320 included participants for hypotheses 14 (after applying our pre-registered exclusion criteria). The entire experiment took around 4 minutes to complete (median completion time in our pilot data: 3.93 minutes). Participants were paid $£ 0.51$ for their participation, equivalent to an hourly wage of $£ 7.78$.

\subsection{Procedure}

A static version of Experiment 2 can be accessed on matanmazor.github.io/termination/experiments/demos/exp2/. Experiment 2 was identical to Experiment 1 with the following exceptions. First, instead of color search trials, we included shape search trials, where the red dot target is present or absent in an array of red squares. Second, to minimize the similarity between conjunction and shape searches, conjunction trials included blue dots and red triangles as distractors. Third, to test participants' explicit metacognition about their visual search behaviour, upon completing the main part of the task participants were presented with the four target-absent displays (shape and conjunction displays with 4 or 8 items), and were asked to sort them from fastest to slowest. Finally, participants answered the question "Have you ever participated in a similar experiment before, where you 
were asked to search for a target item?" Participants who responded 'yes' were asked to tell us more about this previous experiment. This question was included in order to examine whether efficient target-absent search in trial 1 reflects prior experience with similar visual search experiments.

Our pre-registered analysis plan for Experiment 2, including rejection criteria and data preprocessing, was identical to our analysis plan for Experiment 1, and can be accessed in the following link: osf.io/v6mnb. Similar to Exp. 1, here also we time-locked our pre-registration to precede data collection (protocol folder: https://github.com/matanmazor/termination/blob/main/experiments/Experiment2/protocolFolder. zip, protocol sum: ac796c4a9ca81989eec3933f65afbafe697bec4c2473788afb0d3661c177615b, link to relevant lines in experimental code).

\subsection{Results}

On average, participants performed 6.92 practice trials, and $87 \%$ of participants finished the practice part after one block of six trials. Overall mean accuracy in the main task was 0.96 ( standard deviation $=0.06)$. Median reaction time was $644.60 \mathrm{~ms}$ (median absolute deviation $=$ 123.89). In all further analyses, only correct trials with response times between 250 and $1000 \mathrm{~ms}$ are included.

Hypothesis 1 (positive control): Search times in block 2 (target-present) followed the expected pattern, with a steep slope for conjunction search $(M=15.08,95 \%$ CI $[12.34,17.83])$ and a shallow slope for shape search $(M=5.84,95 \%$ CI $[3.90,7.78]$; see middle panel of Fig. 3B). The difference between the slopes was significant $(t(584)=4.98, p<.001 ; \mathrm{d}=0.21)$. 
Hypothesis 2: Our central focus was on results from block 1 (target-absent). Here participants didn't yet have experience with finding the red dot. Similar to the second block, the slope for conjunction search was steep $(M=19.53,95 \%$ CI [16.03, 23.04]). The slope for shape search $(M=8.03,95 \%$ CI $[5.09,10.97])$ was significantly shallower than that of the conjunction search $(t(326)=2.77, p=.006 ; \mathrm{d}=0.15$; see leftmost panel of Fig. 3B), indicating that a processing advantage for detecting the absence of a shape compared to the absence of shape-color conjunction was already in place before experience with target presence.

Moreover, this processing advantage was not different from what is expected based on shape search slope in block 2 (target presence). A conservative estimate for the ratio between target absence and target presence search slopes is 2 (J. M. Wolfe, 1998). Based on this ratio of 2 and the observed target-presence search slope of $6 \mathrm{~ms} /$ item, target absence search slope is expected to be $12 \mathrm{~ms} /$ item, or higher. Indeed, search slope for shape absence was not significantly different from, and numerically lower than, twice the search slope for shape presence as measured in block $2\left(t(548)=-1.16, p=.246 ; \mathrm{BF}_{01}=10.66\right)$.

Hypothesis 3: As in the first block, in the third block the slope for shape search $(M=$ $8.85,95 \%$ CI $[6.67,11.03])$ was significantly different from the the slope for conjunction search $(t(565)=6.02, p<.001 ; \mathrm{d}=0.25)$ and not significantly different from twice the search slope for shape presence $\left(t(653)=1.04, p=.299 ; \mathrm{BF}_{01}=13.29\right.$; see rightmost panel of Fig. 3B).

Hypothesis 4: To quantify a potential learning effect for shape search between blocks 1 and 3, we directly contrasted the search slope for shape search in these two 'target-absent' blocks. We find no evidence for a learning effect $(t(542)=-0.03, p=.974 ; \mathrm{d}=0.00)$. Furthermore, $a$ Bayesian t-test with a scaled Cauchy prior for effect sizes $(r=0.707)$ provided strong evidence against a learning effect $\left(\mathrm{BF}_{01}=20.72\right)$. Like in Experiment 1 , these results are most consistent 
with a prior-knowledge only model (see Fig. 2), in which the efficiency of terminating a search in the absence of a target is not dependent on direct experience with target-present trials.

\subsection{Additional Analyses}

\subsubsection{First trial only.}

As in Exp. 1, here we also extended our pre-registered analysis with an exploratory between-subject analysis, focusing on the first trial of the experiment. Here too, we observed a significant positive search slope for conjunction search (43.65 ms/item, $p<0.001)$, but not for shape search $(9.80 \mathrm{~ms} /$ item, $p=.40)$. The difference in slopes between conjunction and shape, quantified as the interaction between set size and search type in a two-way betwee-subject analysis of variance, was significant $(F(1,781)=4.25$, $M S E=209,989.78, p=.040, \hat{\eta}_{G}^{2}=.005$; see Fig. 4B). This result reveals that efficient recognition of shape absence is already detectable in the very first trial of the experiment.

\subsection{Exploratory analysis: task experience}

At the end of the experiment, participants were asked if they have ever participated in a similar experiment before, where they were asked to search for a target item. 796 out of 887 participants answered 'no' to this question. For those participants, a highly efficient search for a distinct shape in the first trials of the experiment, if found, cannot be due to prior experience of performing a visual search task with similar stimuli. Notably, however, participants who reported having no prior experience with a visual search task still showed efficient search termination for shape distractors $(M=7.32,95 \%$ CI $[4.21,10.43])$, and were significantly more efficient in terminating shape search than conjunction search in the first 4 target-absent trials $(t(296)=$ $2.68, p=.008 ; \mathrm{d}=0.16)$. Efficient search termination for shape search is therefore not dependent on prior visual search trials, neither within the same experiment nor in previous ones. 


\subsection{Exploratory analysis: search time estimates}

A.

Some searches are easier than others. This means that participants find the red dot faster when hidden among some sets of distractors, compared to others.

To the best of your ability, order the following distractor sets from easiest (fastest) to hardest (slowest).

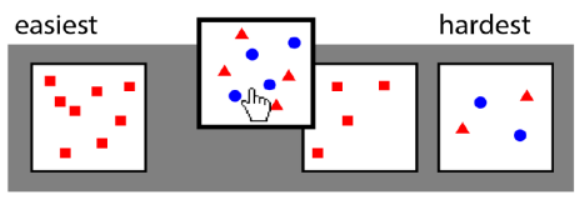

C. Difficulty estimates:

'no insight' group

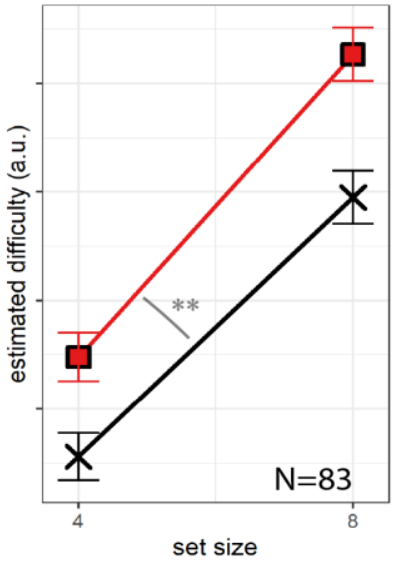

D. Search times: 'no insight' group

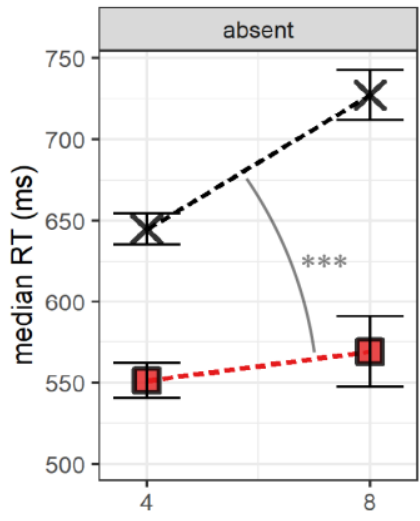

B. Difficulty estimates:

all subjects

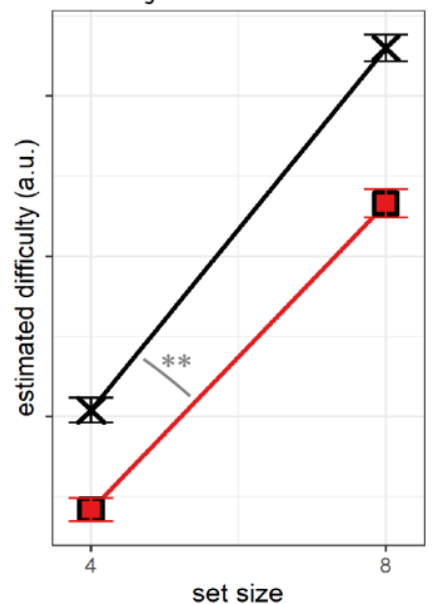

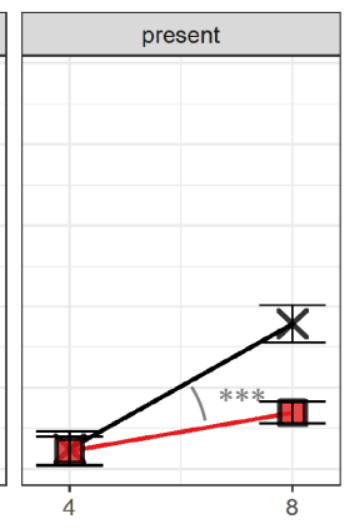

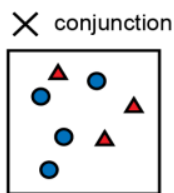

$\square$ shape

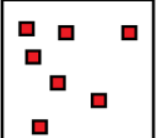

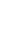


Upon completing the main part of Experiment 2, participants positioned the four search arrays (shape and conjunction searches with 4 or 8 distractors) on a perceived difficulty axis (see Fig. 5A). We used these difficulty ratings to ask whether the advantage for detecting the absence of a distinct shape over the absence of a shape/color conjunction depended on explicit access to metacognitive knowledge about search difficulty. The decision to quit early in shape-absent trials may depend on an internal belief that the target shape would have drawn attention immediately, but this belief may be inaccessible to introspection. If introspective access is not a necessary condition for efficient quitting in visual search, some participants may not be able to reliably introspect about the difficulty of different searches but still be able to quit efficiently in shape search.

For this analysis, we only considered the ratings of participants who engaged with the array-sorting trial, and moved some of the arrays before continuing to the next trial $(\mathrm{N}=789)$. Searches with 8 distractors were rated as more difficult than searches with 4 distractors, in line with the set-size effect $(t(788)=31.62, p<.001 ; d=1.13)$. Furthermore, conjunction searches were rated as more difficult than shape searches $(t(788)=5.11, p<.001 ; \mathrm{d}=0.18)$. Finally, we fitted single-subject linear regression models to the two search types, predicting search-time estimates (the position of each condition on a continuous perceived difficulty scale) as a function of set size. Similar to actual search slopes, these slopes derived from subjective estimates were also shallower for shape than for conjunction search, reflecting a belief that the effect of set size in shape search is not as strong as the effect of set size in conjunction search $(M=6.45,95 \% \mathrm{CI}$ $[2.81,10.08], t(788)=3.48, p=.001 ; d=0.12$; see Fig. $5 B)$.

Subjective search time estimates revealed that by the end of the experiment, the average participant considered the slope of shape search to be shallower than that of conjunction search. 
This suggests that at least some participants had introspective access to their visual search behaviour, either from prior beliefs or from experience in the visual search trials. But were those participants whose estimates reflected a shallow slope for shape search the same ones that were more efficient in detecting the absence of a shape in the display? The slopes of retrospective estimates for shape search were not reliably correlated with actual search slopes for shape absence in block $1(r=.08,95 \%$ CI $[-.06, .22])$ or $2(r=.02,95 \%$ CI $[-.12, .16])$. However, this result should be interpreted carefully in light of the low reliability of single subject estimates that are derived from one trial per cell. Indeed, search slopes for shape absence in blocks 1 and 3 were not reliably correlated themselves $(r=.05,95 \%$ CI $[-.10, .19])$.

To answer this question using a more severe test (Mayo, 2018), we focused on the subset of participants whose difficulty orderings reflected the erroneous belief that shape search was more difficult than conjunction search $(N=83$; see Fig. 5C). If efficient search termination depends on accurate explicit metacognitive knowledge about search efficiency, search termination in this subset of participants is not expected to be more efficient in shape compared to conjunction search, and is even expected to show the opposite pattern. In contrast with this prediction, search slopes for shape-absence trials were shallower than for conjunction-absence trials $\left(M_{d}=12.45,95 \% \mathrm{CI}[5.21,19.69], t(82)=3.42, p=.001 ; \mathrm{d}=0.38\right.$; see Fig. 5D). This indicates that efficient identification of shape absence is not dependent on explicit metacognitive knowledge about search efficiency.

\section{Discussion}

How do people decide that a target is absent from a visual scene? In this study we considered three candidate answers to this question: counterfactual reasoning ("I would have 
detected the target if it were present"), ensemble perception ("I immediately see that the target is missing") and task heuristics ("Based on previous trials, responding now would balance accuracy and response time"). The third option is different from the first two: while a heuristic calibration of a termination rule may shape search behaviour in classic lab-based experiments comprised of many repetitive trials, it is not available to subjects in one-shot searches in their everyday lives, nor is it available to them in the first trial of the experiment.

To isolate the effect of previous trials on search termination, we focused on the first trials of a visual search task, before participants experience finding the target. Across two experiments, we found that no prior experience with color or shape pop-out in previous trials was needed for participants to be able to terminate the search early when a target would have been found immediately. In other words, participants were sensitive to the counterfactual efficiency with which a hypothetical target would have been detected even in the first trials of the experiment. This result rules out a purely heuristic-based account of search termination and suggests that in these first few trials, participants are relying on prior second-order knowledge about visual attention (e.g., 'red pops out,' or 'a dot would catch my attention'), on a pre-attentional identification of target absence via ensemble statistics, or on a combination of the two.

Ensemble perception allows observers to extract summary statistical information from sets of similar stimuli, without directly perceiving any single stimulus (Whitney \& Yamanashi Leib, 2018). According to this account, if participants immediately perceive that the search array comprises only squares, they might not need to rely on any counterfactual thinking or secondorder knowledge about their visual search behaviour to conclude that no circle was present. Importantly, however, for the global statistical property 'the array comprises only squares' to be extracted from a display without representing individual squares, the visual system must 
represent, explicitly or implicitly, that a non-square item would have been detected by the visual system had it been present. This representation can be implemented, for example, as a threshold on curvature-sensitive neurons ('a round object would have induced a higher firing rate in this neuron population'), or more generally as a likelihood function going from polygons to firing patterns ('The perceived input is most likely under a world state where the display includes only polygons').

As an illustration, assume that Sarah, a participant in our experiment, does not know that a red item would immediately catch her attention in an array of blue distractors. Not only can Sarah not report this fact, this knowledge is not represented and cannot influence her cognitive system. Sarah is now searching for a red dot, and sees a uniform array of blue dots. How can she know that she hasn't missed a red dot? In the absence of prior knowledge (explicit or implicit) about search efficiency, Sarah would have to scan the dots one by one before committing to a 'target absent' response. Therefore, whether or not ensemble perception plays a role in absence pop-out the effects we observe are difficult to account for without postulating some form of prior knowledge about search efficiency.

Should this implicit knowledge about visual search be considered metacognitive? Metacognitive knowledge as typically used in contemporary scientific literature refers to explicit knowledge that can be probed with self-reports. As we show in Exp. 2, this type of declarative metacognitive knowledge is not necessary for efficient search termination. At the same time, it is important not to confuse metacognitive knowledge, or metacognitive representations, with behaviour that appears metacognitive from the point of view of the experimenter. Indeed, metalevel representations of one's own cognition can be necessary for some object-level behaviours, and inversely, some behaviours that appear metacognitive may be carried out without a meta- 
level representation at all (Fleming, Dolan, \& Frith, 2012). For example, in their classic article on metamemory, Nelson and Narens (1990) proposed that bona fide metacognitive representations underpin decisions to terminate a memory search in memory recall tasks - a behaviour that is akin to the termination of visual search under investigation here. Similarly, metacognitive knowledge is sometimes measured as an ability to flexibly adapt information gathering thresholds: similar to a decision to terminate a search, the decision to stop gathering more information is widely accepted to be guided by metacognitive factors in developmental (Leckey et al., 2020; Siegel, Magid, Pelz, Tenenbaum, \& Schulz, 2021) and comparative (Watanabe, Grodzinski, \& Clayton, 2014) psychology.

Finally, it is not a requirement for metacognitive knowledge to be accessible for selfreport; metacognitive knowledge was originally assumed by Flavell (1979) to mostly affect cognition without accessing consciousness at all (i.e. without inducing a 'metacognitive experience'). Different aspects of metacognitive monitoring, including an immediate Feeling of Knowing when presented with a problem, have been attributed to implicit metacognitive mechanisms that share a conceptual similarity with the ones described above (Reder \& Schunn, 1996).

This study is the first to examine search termination outside the context of a multi-trial experiment. In answering some questions, it opens new exciting avenues for further research about inference about perceptual absences. First, while we were able to show that efficient search termination in feature search is independent of task experience, we were unable to determine the putative mechanism by which this is achieved. Future studies may seek to isolate the role of ensemble perception by examining absence pop-out in heterogeneous displays (for example, searching for a red dot among blue squares and yellow dots), where decisions about absence 
cannot be based on a direct perception of uniformity. Conversely, to isolate a role for secondorder knowledge, we may be able to find contrast cases where search termination is inefficient due to inaccurate second-order knowledge about search efficiency. Examining termination efficiency in other shallow-slope searches may reveal such dissociations. Finally, here we tested metacognitive beliefs about search efficiency only after engagement with the task, potentially conflating prior knowledge and task experience. Prospective search time estimates would have interfered with our main manipulation but can provide a more direct measure of explicit prior knowledge in future studies.

Our findings complement and extend previous work in which participants had introspective awareness of attentional capture (Adams \& Gaspelin, 2020, 2021): our results suggest that on top of the ability to monitor attention, people (or their visual systems) also hold valid second-order knowledge about attentional processes, that allows them to make predictions and guide their information gathering decisions. A schematic model of attention has been suggested to be implemented in the brains of many animal species, including all mammals and birds, and to facilitate attention control and monitoring (Graziano, 2013). This kind of implicit second-order knowledge, perhaps together with a capacity to extract ensemble statistics from a display, may be crucial for representing the absence of objects. The critical difference between inferring $X$ is absent and simply lacking the belief $X$ is present is a counterfactual belief that $X$ would have been detected, had it been presented (Mazor, 2021; Mazor \& Fleming, 2020). In turn, studying the processes underpinning efficient inference about absence can shed light on the role of higher-order representations in perception - because such counterfactual beliefs rest on representing, perhaps implicitly, how one's own perceptual system might respond under various conditions. 


\subsection{Conclusion}

Our findings reveal that efficient search termination in the absence of a feature is independent of task experience and of explicit metacognitive knowledge about search efficiency. Search times in the very first trials of the experiment show the same qualitative response time patterns as those commonly obtained in typical (few subjects/many trials) visual search experiments. Given that no target was present in these trials, participants' search termination strategy must have been sensitive to the counterfactual likelihood of them finding the target, had it been present. In Experiment 2 we showed that this knowledge about search difficulty was often accessible to report, but that this was not a necessary condition for efficient search termination. We conclude that efficient inference about absence is critically dependent on implicit secondorder knowledge about visual search, ensemble statistics, or a combination of both.

\section{$5 \quad$ References}

Adams, O. J., \& Gaspelin, N. (2020). Assessing introspective awareness of attention capture. Attention, Perception, \& Psychophysics, 1-13.

Adams, O. J., \& Gaspelin, N. (2021). Introspective awareness of oculomotor attentional capture. Journal of Experimental Psychology: Human Perception and Performance.

Aust, F., \& Barth, M. (2020). papaja: Create APA manuscripts with R Markdown. Retrieved from https://github.com/crsh/papaja

Champely, S. (2020). Pwr: Basic functions for power analysis. Retrieved from https://CRAN.Rproject.org/package $=$ pwr 
Chun, M. M., \& Wolfe, J. M. (1996). Just say no: How are visual searches terminated when there is no target present? Cognitive Psychology, 30(1), 39-78.

D’Zmura, M. (1991). Color in visual search. Vision Research, 31(6), 951-966.

Ekstrøm, C. T. (2019). MESS: Miscellaneous esoteric statistical scripts. Retrieved from https://CRAN.R-project.org/package=MESS

Flavell, J. H. (1979). Metacognition and cognitive monitoring: A new area of cognitivedevelopmental inquiry. American Psychologist, 34(10), 906.

Fleming, S. M., Dolan, R. J., \& Frith, C. D. (2012). Metacognition: Computation, biology and function. The Royal Society.

Graziano, M. S. (2013). Consciousness and the social brain. Oxford University Press.

Hulleman, J., \& Olivers, C. N. (2017). The impending demise of the item in visual search. Behavioral and Brain Sciences, 40.

Ishibashi, K., Kita, S., \& Wolfe, J. M. (2012). The effects of local prevalence and explicit expectations on search termination times. Attention, Perception, \& Psychophysics, 74(1), 115-123.

Leckey, S., Selmeczy, D., Kazemi, A., Johnson, E. G., Hembacher, E., \& Ghetti, S. (2020). Response latencies and eye gaze provide insight on how toddlers gather evidence under uncertainty. Nature Human Behaviour, 4(9), 928-936.

Mayo, D. G. (2018). Statistical inference as severe testing. Cambridge: Cambridge University Press. 
Mazor, M. (2021). Inference about absence as a window into the mental self-model.

Mazor, M., \& Fleming, S. M. (2020). Distinguishing absence of awareness from awareness of absence. Philosophy and the Mind Sciences, 1(II).

Mazor, M., \& Fleming, S. M. (2021, February 18). Metacognitive contributions to search termination. Retrieved from osf.io/fyjcp

Mazor, M., Mazor, N., \& Mukamel, R. (2019). A novel tool for time-locking study plans to results. European Journal of Neuroscience, 49(9), 1149-1156.

Moran, R., Zehetleitner, M., Müller, H. J., \& Usher, M. (2013). Competitive guided search: Meeting the challenge of benchmark RT distributions. Journal of Vision, 13(8), 24-24.

Morey, R. D., \& Rouder, J. N. (2018). BayesFactor: Computation of bayes factors for common designs. Retrieved from https://CRAN.R-project.org/package=BayesFactor

Navarro, D. (2015). Learning statistics with $r$ : A tutorial for psychology students and other beginners. (Version 0.5). Adelaide, Australia: University of Adelaide. Retrieved from http://ua.edu.au/ccs/teaching/lsr

Nelson, T. O., \& Narens, L. (1990). Metamemory: A theoretical framework and new findings. In Psychology of learning and motivation (Vol. 26, pp. 125-173). Elsevier.

Ooms, J. (2014). The jsonlite package: A practical and consistent mapping between JSON data and r objects. arXiv:1403.2805 [Stat.CO]. Retrieved from https://arxiv.org/abs/1403.2805

R Core Team. (2019). R: A language and environment for statistical computing. Vienna, Austria: R Foundation for Statistical Computing. Retrieved from https://www.R-project.org/ 
Reder, L. M., \& Schunn, C. D. (1996). Metacognition does not imply awareness: Strategy choice is governed by implicit learning and memory.

Siegel, M. H., Magid, R. W., Pelz, M., Tenenbaum, J. B., \& Schulz, L. E. (2021). Children’s exploratory play tracks the discriminability of hypotheses. Nature Communications, 12(1), $1-9$.

Treisman, A. (2006). How the deployment of attention determines what we see. Visual Cognition, 14(4-8), 411-443.

Treisman, A. M., \& Gelade, G. (1980). A feature-integration theory of attention. Cognitive Psychology, 12(1), 97-136.

Watanabe, A., Grodzinski, U., \& Clayton, N. S. (2014). Western scrub-jays allocate longer observation time to more valuable information. Animal Cognition, 17(4), 859-867.

Whitney, D., \& Yamanashi Leib, A. (2018). Ensemble perception. Annual Review of Psychology, $69,105-129$.

Wickham, H. (2016). ggplot2: Elegant graphics for data analysis. Springer-Verlag New York. Retrieved from https://ggplot2.tidyverse.org

Wickham, H., François, R., Henry, L., \& Müller, K. (2020). Dplyr: A grammar of data manipulation. Retrieved from https://CRAN.R-project.org/package=dplyr

Wickham, H., \& Henry, L. (2020). Tidyr: Tidy messy data. Retrieved from https://CRAN.Rproject.org/package=tidyr 
Wilke, C. O. (2019). Cowplot: Streamlined plot theme and plot annotations for 'ggplot2'. Retrieved from https://CRAN.R-project.org/package=cowplot

Wolfe, J. (2021). Guided search 6.0: An updated model of visual search. Psychonomic Bulletin \& Review.

Wolfe, J. M. (1998). What can 1 million trials tell us about visual search? Psychological Science, 9(1), 33-39.

Wolfe, J. M. (2012). When do i quit? The search termination problem in visual search. The Influence of Attention, Learning, and Motivation on Visual Search, 183-208.

Wolfe, J. M., \& Gray, W. (2007). Guided search 4.0. Integrated Models of Cognitive Systems, $99-119$.

Wolfe, J. M., \& Horowitz, T. S. (2017). Five factors that guide attention in visual search. Nature Human Behaviour, 1(3), 1-8.

Wolfe, J. M., Horowitz, T. S., \& Kenner, N. M. (2005). Rare items often missed in visual searches. Nature, 435(7041), 439-440. 
Appendix

\section{A Effect of RT-based trial exclusion}

Our pre-registered exclusion criterion for particularly slow (>1000 ms) and fast (<250 ms) trials resulted in the exclusion of a non-negligible number of trials per participant (more than two out of 12 trials on average).

\section{Experiment 1}

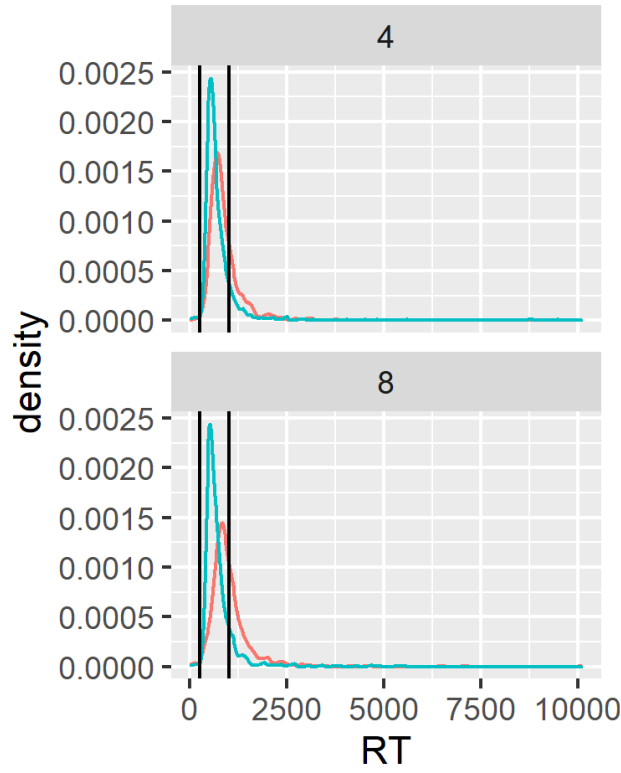

Search type $\square$ conjunction $\square$

\section{Experiment 2}

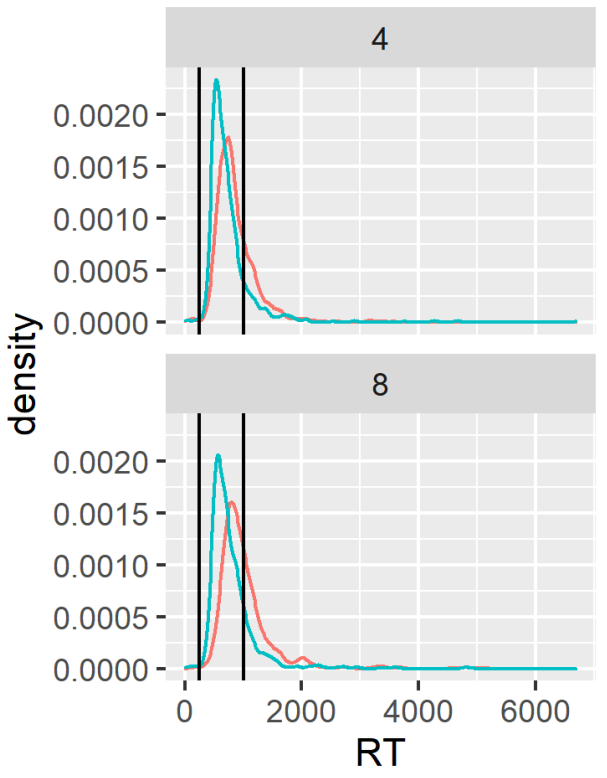

search type conjunction

Figure A1. RT histograms in the first block (first four trials) of Exp. 1 and 2 as a function of search type and set size. Our pre-registered analysis included only trials between the two vertical lines, corresponding to 250 and $1000 \mathrm{~ms}$.

To test the robustness of our findings to other RT-based exclusion criteria, we report here all pre-registered analyses, this time without excluding trials based on response time. Relaxing the RT-based exclusion criterion did not affect the results of most of our four pre-registered 
analyses, with the following exceptions: slopes for conjunctions slopes were now generally higher, and in Experiment 2, block 3, shape slope was not significantly different from the slope for conjunction search. Importantly, even when including these trials, shape slope was significantly different from conjunction slope in block 1. Furthermore, like in the original report, here also we find no learning effect between blocks 1 and 3.

\section{A.1 Experiment 1. Hypothesis 1 (positive control): Search times in block 2} (target-present) followed the expected pattern, with a steep slope for conjunction search $(M=$ $17.27,95 \% \mathrm{CI}[12.38,22.15])$ and a shallow slope for color search $(M=2.90,95 \%$ CI $[-0.59$, 6.39]). The difference between the slopes was significant $(t(891)=4.25, p<.001)$.

Hypothesis 2: Similar to the second block, the slope for the conjunction search was steep $(M=35.99,95 \%$ CI $[27.56,44.43])$ whereas the slope for color search was shallow $(M=$ $-1.03,95 \%$ CI $[-8.43,6.37])$. The average search slope for color search in this first block was significantly different from that of the conjunction search $(t(874)=6.36, p<.001)$, indicating that a color-absence pop-out is already in place prior to direct task experience.

Hypothesis 3: In the third block color search slope was shallow $(M=1.91,95 \%$ CI $[-1.83,5.66])$, and significantly different from the conjunction search slope $(t(964)=7.92$, $p<.001)$

Hypothesis 4: We find no evidence for a learning effect $(t(996)=-0.86, p=.389)$. Furthermore, a Bayesian t-test with a scaled Cauchy prior for effect sizes ( $\mathrm{r}=0.707)$ provided strong evidence in favour of the absence of a learning effect $\left(\mathrm{BF}_{01}=19.35\right)$.

Hypothesis 5: The change in slope between blocks 1 and 3 was similar for color and conjunction search $(M=-9.31,95 \%$ CI $[-21.70,3.09], t(745)=-1.47, p=.141)$. 


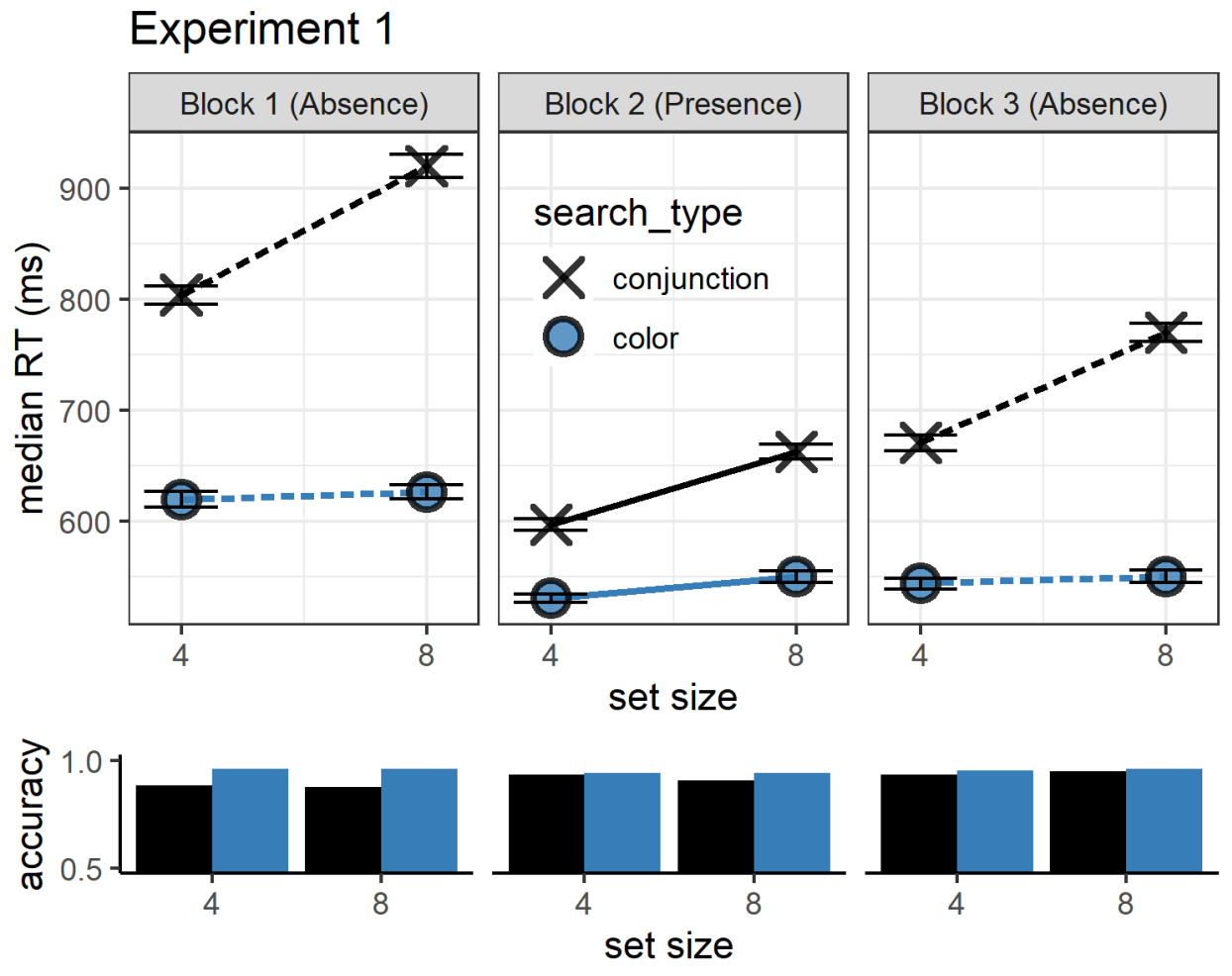

Figure A2. Results from Experiment 1 without RT-based trial exclusion

\section{A.2 Experiment 2. Hypothesis 1 (positive control): Search times in block 2}

(target-present) followed the expected pattern, with a steep slope for conjunction search $(M=$ $21.87,95 \%$ CI $[15.55,28.19])$ and a shallow slope for shape search $(M=1.99,95 \%$ CI $[-3.81$, 7.79]). The difference between the slopes was significant $(t(680)=5.39, p<.001)$.

Hypothesis 2: Also in the first block, the slope for conjunction search was steep $(M=$ $34.71,95 \%$ CI $[27.71,41.71])$, and the slope for shape search shallow $(M=9.68,95 \% \mathrm{CI}$ $[3.13,16.23])$. The average search slope for shape search in this first block was significantly different from that of the conjunction search $(t(701)=5.02, p<.001)$.

Hypothesis 3: In the third block the slope for shape search was numerically lower, but not significantly different than the slope for conjunction search $(t(751)=0.81, p=.419)$. 
Hypothesis 4: To quantify a potential learning effect for shape search between blocks 1

and 3, we directly contrasted the search slope for shape search in these two 'target-absent' blocks. We find no evidence for a learning effect $(t(751)=-1.03, p=.303)$. Furthermore, a Bayesian t-test with a scaled Cauchy prior for effect sizes $(r=0.707)$ provided strong evidence against a learning effect $\left(\mathrm{BF}_{01}=14.37\right)$.

\section{Experiment 2}
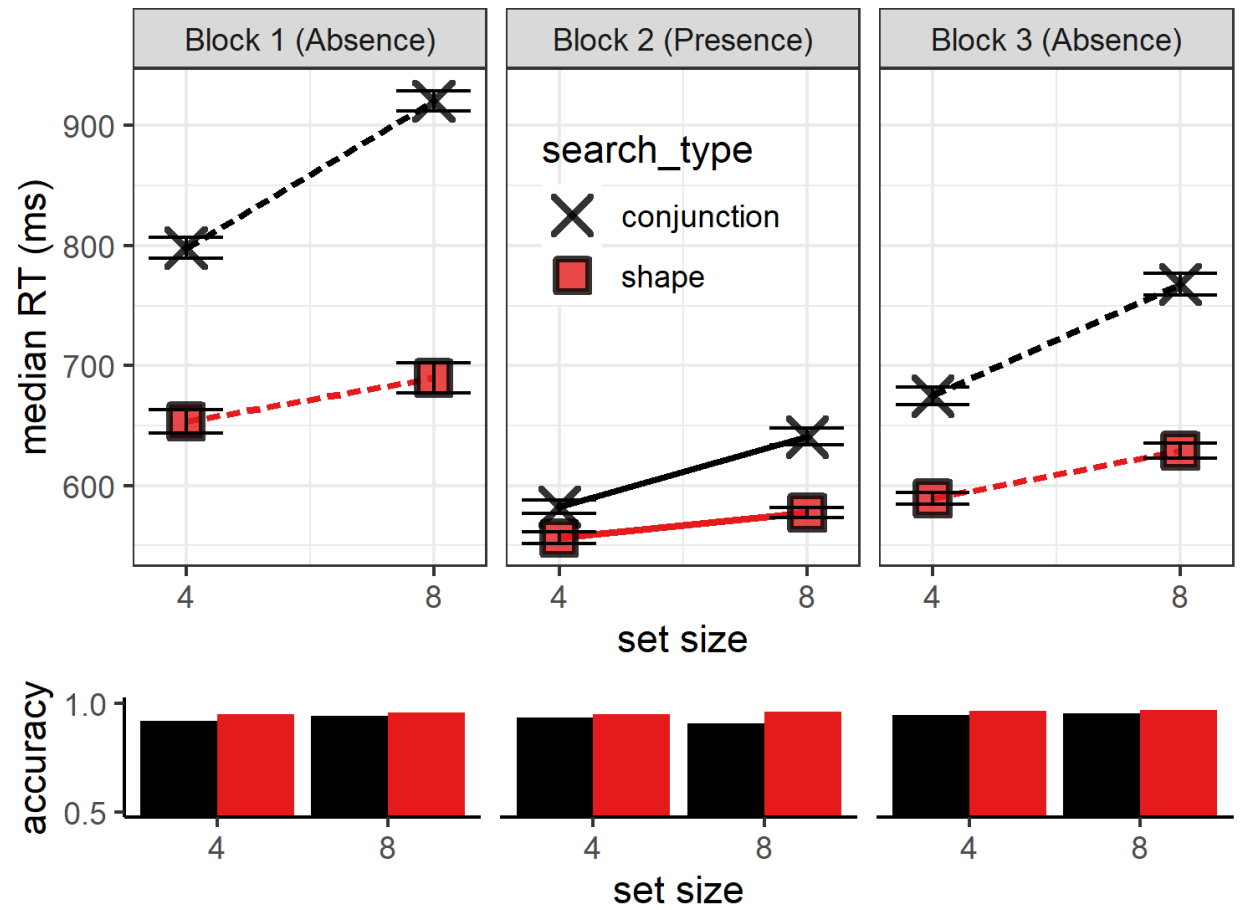

Figure A3. Results from Experiment 1 without RT-based trial exclusion 
B practice trials

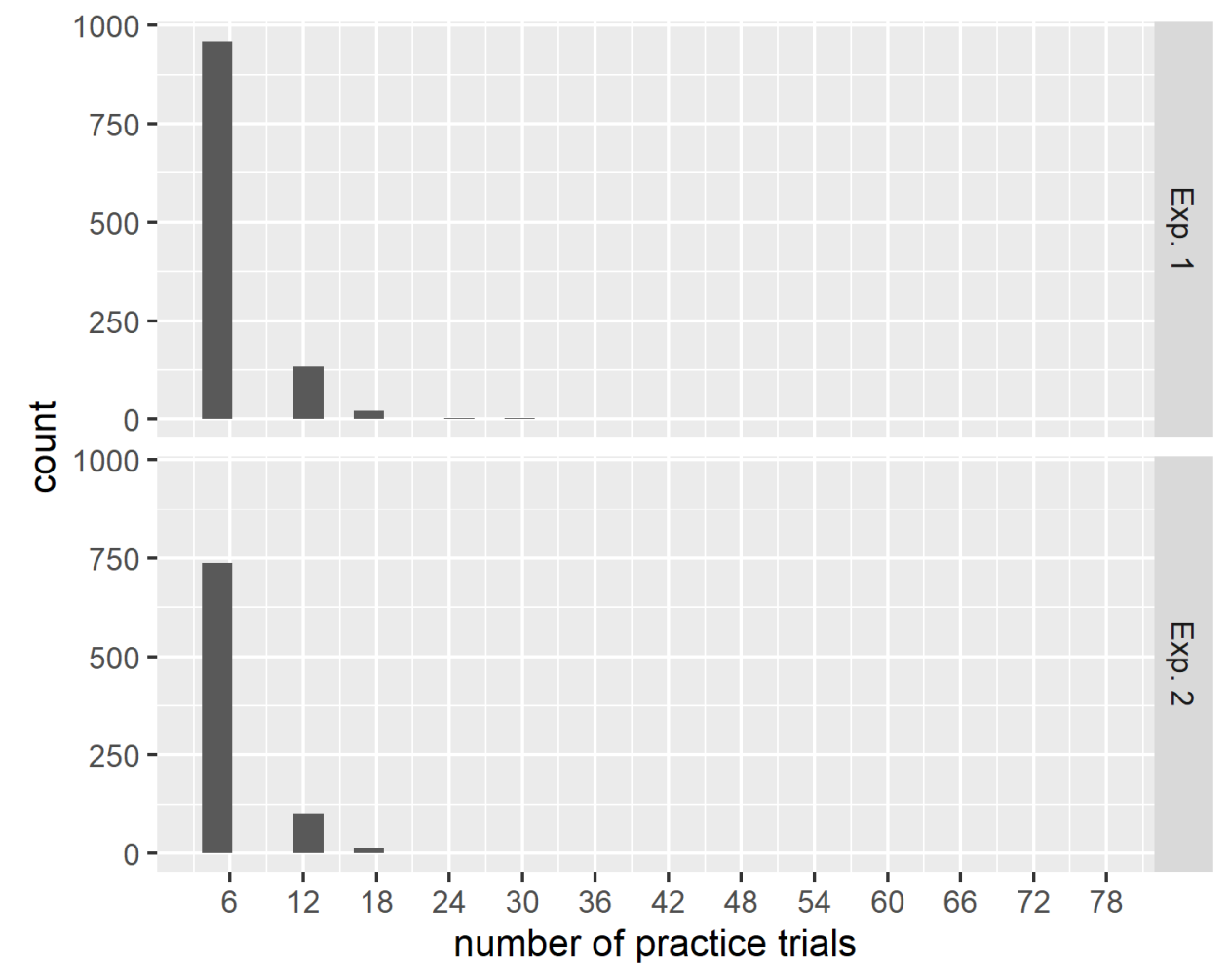

Figure B1. Distribution of the number of practice trials performed before reaching the desired accuracy level 


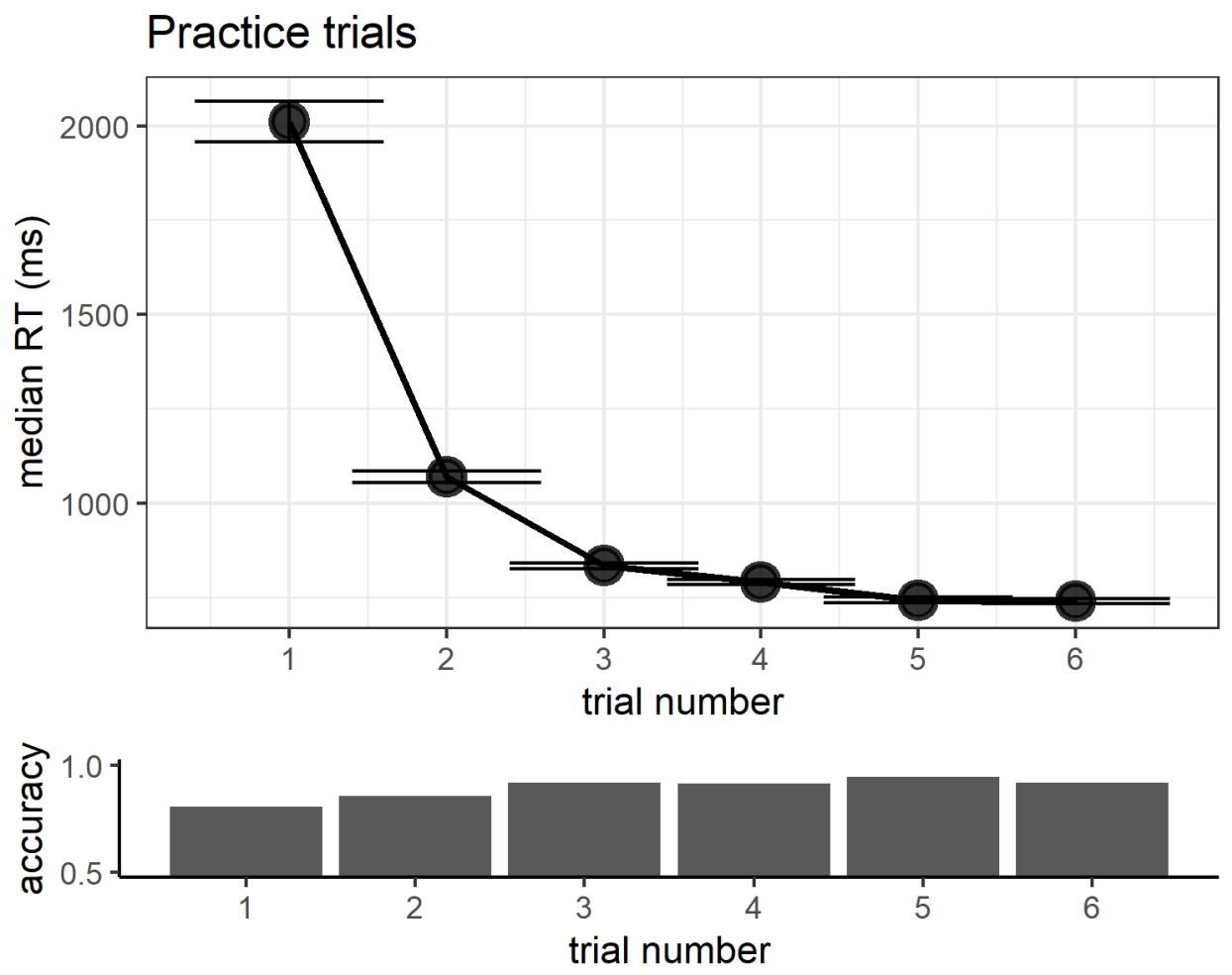

Figure B2. Upper panel: response time as a function of serial position within the first practice block, correct trials only. Lower panel: accuracy as a function of serial position within the first practice block

\section{Trial order correction}

We wanted to rule out a potential concern that our results are driven by an artifact of the pre-registered trial order correction (see Methods). As we show in the main text, similar findings are obtained when analyzing only the first trial of each participant - an analysis that does not involve any order correction. In addition, here we repeated our pre-registered analyses (specifically, $\mathrm{H} 2$ in both Experiments) on raw search times.

Similar to our main results, in Exp. 1 conjunction absence search slope was steep $(M=$ $18.09,95 \%$ CI $[14.01,22.18])$, color absence was shallow $(M=-0.35,95 \%$ CI $[-3.21$, 
2.51]), and the average search slope for color was significantly different from that of conjunction $(t(413)=6.02, p<.001)$. In Exp. 2, conjunction absence search slope was steep $(M=18.40$ 95\% CI $[14.39,22.41])$, shape absence was shallow $(M=7.64,95 \%$ CI $[4.37,10.90])$, and the average search slope for shape was significantly different from that of conjunction $(t(326)=$ $2.03, p=.043)$. In conclusion, our results remain robust to whether we actively control for serial order effects, or allow them to average out by trial order randomization.

\section{Pop-out as a search slope below $10 \mathrm{~ms} /$ item}

In our pre-registration document, pop-out search was defined as a search that produces a slope significantly lower than $10 \mathrm{~ms} /$ item. We subsequently realized that this definition was flawed: empirical distributions of search slopes suggest there is no hard line between 'parallel' and 'serial' searches (J. M. Wolfe, 1998). We therefore omitted these tests from the main text, and present them here instead for completeness.

\section{D.1 Experiment 1.}

D.1.1 Block 1 (target absence).

Color-absence search slope was significantly below $10(t(886)=-7.51, p<.001$; $\mathrm{d}=0.25)$.

D.1.2 Block 2 (target presence). Color-presence search slope was significantly below $10(t(961)=-6.69, p<.001 ; \mathrm{d}=0.22)$.

D.1.3 Block 3 (target absence). Color-absence search slope was significantly below $10(t(979)=-7.98, p<.001 ; \mathrm{d}=0.25)$.

\section{D.2 Experiment 2.}

D.2.1 Block 1 (target absence). 
Shape-absence search slope was not significantly below $10(t(608)=-1.31, p=.095$; $\mathrm{d}=0.05)$.

D.2.2 Block 2 (target presence). Shape-presence search slope was significantly below $10(t(754)=-4.21, p<.001 ; \mathrm{d}=0.15)$.

D.2.3 Block 3 (target absence). Shape-absence search slope was not significantly below $10(t(723)=-1.03, p=.151 ; \mathrm{d}=0.04)$.

\section{E Learning effect for conjunction search}

In our pre-registered hypotheses we focused on feature search slopes, using conjunction search as a baseline condition. Our assumption was that conjunction search slopes should not change between blocks 1 and 3, and that any learning effect would be evident in feature search slopes instead. Indeed, we find no effect of block number on conjunction search slopes in Exp. 1 $(M=2.08,95 \%$ CI $[-2.84,6.99], t(385)=0.83, p=.407)$ nor in Exp. $2(M=-2.15,95 \%$ CI $[-6.96,2.65], t(344)=-0.88, p=.379)$. 